\title{
Originals
}

\section{Sorbinil does not prevent galactose-induced glomerular capillary basement membrane thickening in the rat}

\author{
A. Das, R.N. Frank and N.L.Zhang \\ Kresge Eye Institute, Wayne State University School of Medicine, Detroit, Michigan, USA
}

\begin{abstract}
Summary. We investigated the role of the polyol pathway in the pathogenesis of glomerular basement membrane thickening in galactosaemic rats, an animal model that develops basement membrane lesions comparable to those of human diabetic subjects. Normotensive Wistar-Kyoto rats fed a $30 \%$ galactose diet for nine months developed significant glomerular basement membrane thickening by comparison with rats on a control test diet $(p=0.008)$. However, addition of an aldose reductase inhibitor, sorbinil $(250 \mathrm{mg} / \mathrm{kg}$ diet), to the galactose diet did not prevent the increase in glomerular basement membrane thickness. Furthermore, by using a quantitative electron microscopic immunogold technique, we examined biochemical alterations in the composition of glomerular basement membranes in this animal model. The labelling density (comparable to relative concentration) of collagen type IV in thickened glomerular base-
\end{abstract}

ment membranes of galactosaemic animals was significantly increased by comparison to those of control rats $(p=0.015)$. However, there was no significant difference in labelling densities of laminin and heparan sulfate proteoglycan core protein of these animals. Thus, our results indicate that an increase in glomerular basement membrane thickness accompanied by an increase in the labelling density of collagen type IV occurs in the galactosaemic rats, but this thickening is not prevented by sorbinil at the dose used in this experiment. Our study raises the strong possibility that glomerular basement membrane thickening in galactosaemic rats may not be due to excessive polyol pathway activity.

Key words: Basement membrane, galactosaemia, aldose reductase inhibitors, glomerulus, microvessels.
The polyol pathway has been implicated in the development of various complications of diabetes such as cataract, retinopathy and neuropathy $[1,2]$. Whether such a mechanism plays an important role in the pathogenesis of diabetic nephropathy remains controversial. The enzyme, aldose reductase has been immunocytochemically localized in glomerular podocytes [3] and cultured mesangial cells [4], and elevated concentrations of galactitol in the renal cortex of galactosaemic rats [5] and sorbitol in the renal papillae in diabetes [6] have been described. Although several investigators have shown that administration of aldose reductase inhibitors prevents renal hyperperfusion and increase in glomerular filtration rate in diabetic rats [7-9], one report failed to show such effect in these animals [10]. However, aldose reductase inhibition does not prevent glomerular basement membrane (GBM) thickening in diabetic rats [11-13].

To examine further the relationship of the polyol pathway to diabetic nephropathy, we fed rats a diet containing $30 \%$ by weight galactose for nine months. These animals develop thickened retinal and cerebral capillary basement membranes comparable to those found in diabetic animals and humans [14-16]. Glomerular hyperperfusion has been reported in the galactosaemic rats [17], and GBM thickening has been described in long-term galactosaemic dogs [18]. Both retinal and cerebral capillary basement membrane thickening in galactosaemic rats are prevented by the simultaneous administration of aldose reductase inhibitors [14-16]. One group of investigators reported prevention of an important early lesion of diabetic retinopathy, capillary pericyte loss, by giving aldose reductase inhibitors to galactosaemic dogs [19]. However, this has not been confirmed by others [20]. In the present study, we examined the GBMs of galactosaemic rats by quantitative electron microscopy in the presence and absence of an aldose reductase inhibitor. Using a quantitative electron microscopic immunocytochemical technique, we investigated the relative concentrations of various macromolecular components of GBMs in these animals. In a previous study, we used this technique to demonstrate increases in 
collagen type IV and laminin in retinal capillary basement membranes in galactosaemic rats, and the prevention of these changes in galactosaemic rats that were simultaneously fed an aldose reductase inhibitor [21].

\section{Materials and methods}

\section{Animals}

Fifteen normotensive Wistar-Kyoto female rats (Taconic Farms, Germantown, NY, USA) at six weeks of age (body weight about $100 \mathrm{~g}$ ) were used in this study and maintained on different diets for nine months. The animals were divided into three groups (five animals in each group), and each group was given a different type of diet: a) the standard Ralston Purina (Purina Mills, Richmond, Ind., USA) test diet (carbohydrate, 61\%; protein, 19\%; fat, 10\%; fiber, $3 \%$; vitamins and minerals) (control animals), b) a diet in which the major carbohydrate source, dextrin was replaced by $\mathrm{D}$-galactose to $30 \%$ by weight of the diet, and c) a diet of $30 \%$ galactose-plus-sorbinil (250 mg/kg diet; a gift from Dr. N. Hutson, Pfizer Central Research, Groton, Conn., USA). The food consumption of these rats was approximately $15 \mathrm{~g}$ per day per animal, and each animal in the sorbinil treated group received about $3 \mathrm{mg}$ sorbinil per day.

\section{Tissue fixation and processing}

Animals were maintained on the dietary protocols for nine months, and then killed by barbiturate anaesthesia followed by bilateral pneumothorax. The kidneys from these animals were excised, and small pieces of cortical tissues from the upper poles of the right kidneys fixed in $3 \%$ formaldehyde and $0.5 \%$ glutaraldehyde in $0.1 \mathrm{~mol} / \mathrm{l}$ phosphate buffer, $\mathrm{pH} 7.4$, for $2 \mathrm{~h}$ at $4^{\circ} \mathrm{C}$, while the rest of the kidney tissues were frozen for other biochemical assays. Tissues were washed in $0.5 \mathrm{~mol} / 1 \mathrm{NH}_{4} \mathrm{Cl}$ in buffer for $1 \mathrm{~h}$, dehydrated in increasing concentrations of methanol and embedded in LR White (Polysciences Inc., Warrington, $\mathrm{Pa}$, USA). Polymerization was carried out in tightly capped gelatin capsules at $45^{\circ} \mathrm{C}$ for three days. One micron thick sections were cut, and random selection was done to find the centermost intact glomeruli in the block [22]. Ultrathin sections were then obtained, mounted on 200-mesh nickel grids, and stored in the desiccator until further use for immunogold staining.

\section{Primary antibodies}

The rabbit antiserum against mouse Engelbreth-Holm-Swarm (EHS) tumour collagen type IV (BioDesign, Kennenbunkport, Me, USA) has been characterized by RIAs as described elsewhere [23]. The specificity of the rabbit antiserum against mouse EHS tumour laminin (Polysciences) has been determined in our laboratory by immunoblotting. The rabbit antiserum against heparan sulfate proteoglycan (HSPG) extracted from EHS tumour (a gift from Dr. J.Hassell, Department of Ophthalmology, University of Pittsburgh, Pittsburgh, Pa, USA), was passed through a column of laminin bound to Sepharose CL-4B, and then adsorbed to a second column of proteoglycan and finally eluted [24]. This antibody was specific for the HSPG protein core, and did not react with either laminin or collagen type IV.

\section{Electron microscopic immunocytochemistry}

Ultrathin sections on grids were floated on drops of water for $5 \mathrm{~min}$, and then transferred to drops of the blocking reagent, containing 1\% bovine serum albumin (BSA) (Janssen Pharmaceutica,
Piscataway, NJ, USA) and 5\% normal goat serum (NGS) (Vector Laboratories Inc., Burlingame, Calif., USA) in $20 \mathrm{mmol} / 1$ Tris buffered $0.9 \% \mathrm{NaCl}$ solution (TBS), $\mathrm{pH} 7.4$ for $30 \mathrm{~min}$ at room temperature. Grids were then incubated on drops of specific primary antibodies diluted in $20 \mathrm{mmol} / \mathrm{l}$ TBS containing $1 \% \mathrm{BSA}$ and $1 \%$ NGS, for $2 \mathrm{~h}$. The following dilutions of primary antibodies were used: collagen type IV $(1: 10)$, laminin $(1: 5)$ and HSPG core protein $(1: 15)$. After several washes with TBS, containing $0.1 \%$ BSA, grids were floated on drops of goat anti-rabbit IgG labelled with 10 or $15 \mathrm{~nm}$ gold particles (Janssen) diluted $1: 20$ in TBS, pH 8.2, containing $1 \%$ BSA, for $1 \mathrm{~h}$. After washes with TBS and distilled water, sections were further stained with uranyl acetate and lead citrate, and examined in a Philips 300 transmission electron microscope.

Sections incubated with non-immune sera served as controls. All the tissues from the three different groups of animals were fixed and processed on the same day in identical ways. For each individual antibody, all the sections were immunostained on the same day in the same way. This was very important for our quantitative immunocytochemical study for comparison purposes. After several trials of different concentrations of primary and secondary antibodies, the optimal concentrations were worked out so that there was maximal specific staining on the basement membranes and minimal background staining.

\section{Quantitative morphometry}

For each antibody staining, 20 capillaries from each group of animals (four capillaries from each animal) were photographed at the same magnification of $\times 5,900$, and enlarged to the final magnification of approximately $\times 16,225$ during printing. From each animal, two blocks of kidney tissues were chosen at random, and in each block two capillaries in the centermost glomerulus were chosen. Only those capillaries, whose major axes did not differ in length by more than a factor of two, and with sharply delineated borders of basement membranes, were photographed in this study. As the GBM width is highly influenced by section angle, the sharpness of both basement membrane borders served as a criterion for perpendicularity to the plane of section [25]. The exact magnification of the photographs was calculated using electron micrographs of a diffraction grating replica (2,160 lines per $\mathrm{mm}$; Polysciences). Quantitative morphometry was done on these prints by an observer, unaware of the groups of animals from which they were chosen. An Apple IIe microcomputer, equipped with BioQuant image analysis software (R \& M Biometrics, Nashville, Tenn., USA) and a HiPad digitizer tablet (Houston Instruments, Division of Bausch and Lomb, Austin, Tex., USA) was used for quantitation.

By outlining the borders of basement membranes, basement membrane area (BMA) and basement membrane perimeter (BMP; taken by summing both the inner and outer perimeters of all basement membranes) were measured for each capillary. The beginning of the mesangium was determined on the criterion of disappearance of parallelism between the endothelial and epithelial cells [26], and the GBM interposed between the mesangium and epithelial cells was excluded from measurement. Thus, only the peripheral glomerular capillary basement membrane was included for quantitation. Next, the basement membrane length (BML) and the relative basement membrane thickness (BMT) were determined as follows:

$\mathrm{BML}=\mathrm{BMP} / 2$

$\mathrm{BMT}=\mathrm{BMA} / \mathrm{BML}$

The total number of individual gold particles distributed over the basement membranes for each antibody was counted. The labelling density for each antigen could then be expressed as the number of gold particles per unit area of basement membrane. Labelling densities for each antigen were then compared among these groups of animals. Occasionally, clusters of gold particles were found on the basement membranes, and these were excluded from counting. 


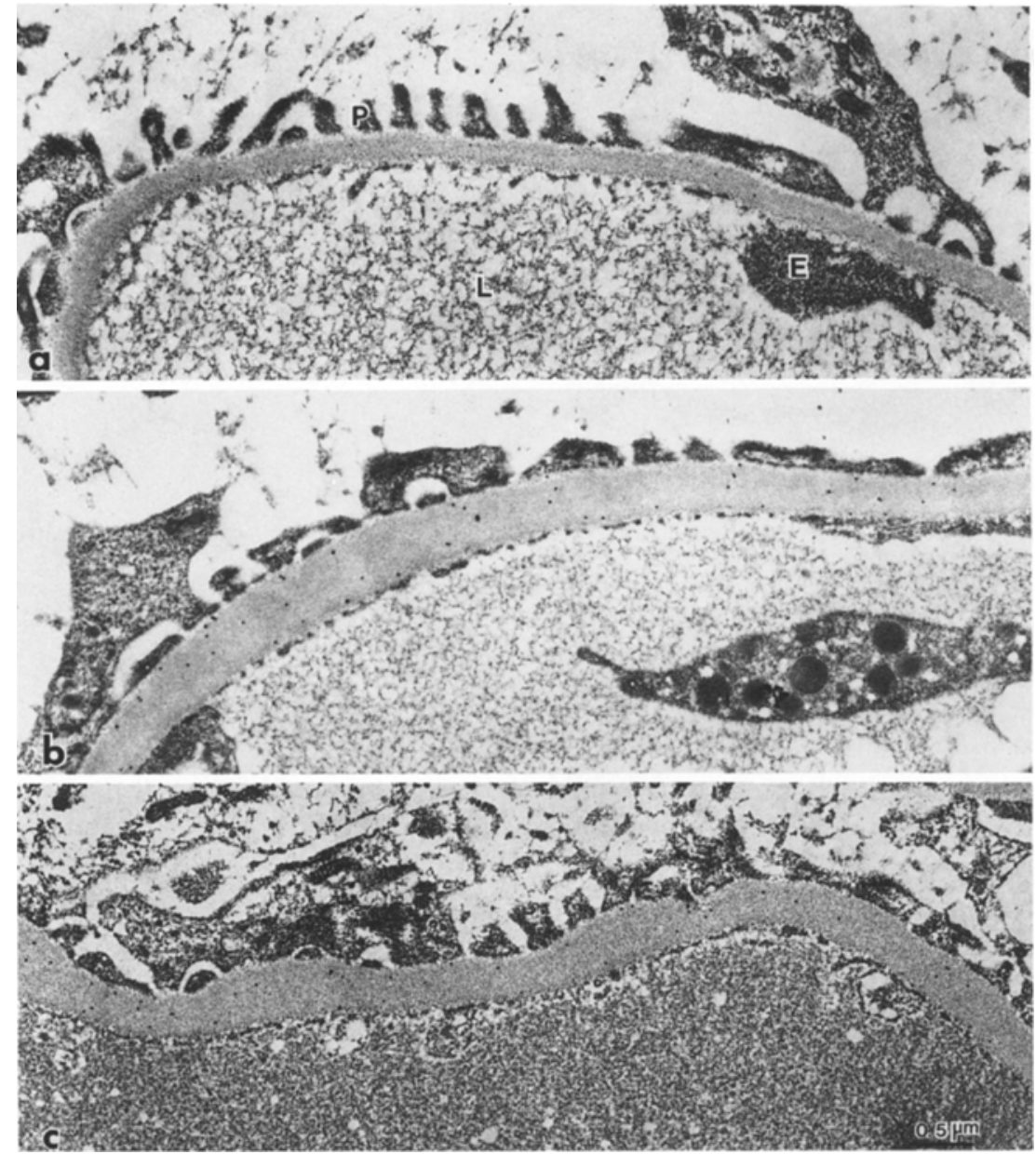

Fig. 1a-c. Immunogold labelling for collagen type $\mathrm{IV}$ in glomerular basement membranes in rats on a control diet, $\mathbf{b}$ galactose diet, and c galactose plus sorbinil diet. Antigenic sites are specifically labelled with $15 \mathrm{~nm}$ size gold particles. $\mathbf{P}=$ podocyte $; \mathrm{E}=$ endothelium; $\mathrm{L}=$ lumen of capillary. Bar, $0.5 \mu \mathrm{m}$

\section{Statistical analysis}

As four capillaries were chosen per animal, the individual measurements were averaged for each animal, and then these values were averaged for five animals in each experimental group to obtain the means and SD used for the final calculation of significance. The unpaired Student's $t$-test was used to determine the significance of differences among the different groups of animals.

\section{Results}

\section{Basement membrane thickness}

Galactose-fed rats showed a significant increase in basement membrane thickness of glomerular capillaries (both peripheral and central) compared to rats that were on the control diet ( $p=0.008$; Table 1; Figs. 1-3). The thickening of the basement membranes was evenly distributed. In the galactose plus sorbinil-treated group, GBM thickness was not significantly different from that in the galactose-fed group (Table 1) (Figs. 1-3).

\section{Labelling densities of basement membrane components}

Sections stained with collagen type IV antibody showed specific localization of gold particles throughout the thickness of the glomerulat basement membranes
(Fig. 1 a-c). The intensity of labelling in the mesangium was always much higher than that in the basement membranes. The labelling density for collagen type IV in the galactose-fed animals was significantly higher than that in the control group ( $p=0.015)$ (Table 2). Capillaries in the sorbinil-treated animals did not show any significant difference in labelling density for collagen type IV in comparison with capillaries from the galactose-fed animals.

The localization of laminin in the GBMs was also very specific, and the labelling was found throughout the thickness of basement membranes (Figs.2a-c). The labelling density for laminin in galactose-fed animals was not significantly different from that in either the control animals or the sorbinil-treated animals (Table 2).

Table 1. Glomerular basement membrane thickness (GBMT) in three groups of animals, each group composed of five animals (mean \pm SD in $\mathrm{nm}$ )

\begin{tabular}{ll}
\hline Diet & GBMT \\
\hline Control & $314.0 \pm 22.62$ \\
Galactose & $416.6 \pm 61.63^{\mathrm{b}}$ \\
Galactose + Sorbinil & $389.4 \pm 20.17^{\mathrm{a}, \mathrm{c}}$ \\
\hline
\end{tabular}

${ }^{a}$ No significant difference from galactose value; ${ }^{b}$ differs from control value $(p=0.008) ;{ }^{c}$ differs from control value $(p<0.001)$ 


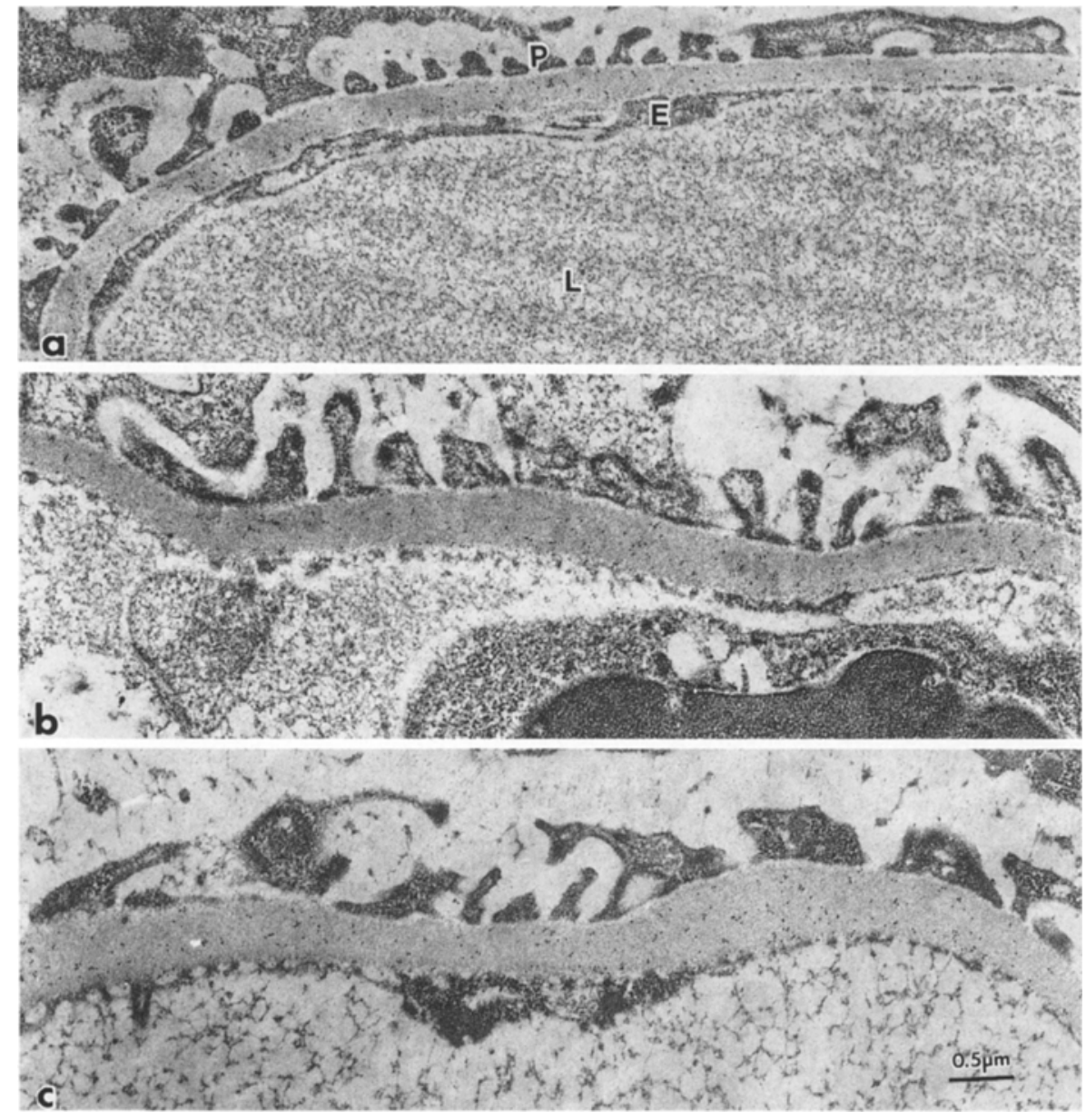

Fig.2a-c. Immunogold labelling for laminin in glomerular basement membranes in rats on a control diet, b galactose diet, and $\mathbf{c}$ galactose plus sorbinil diet. Antigenic sites are labelled with $10 \mathrm{~nm}$ size gold particles.

$\mathrm{P}=$ podocyte $\mathrm{E}=$ endothelium; $\mathrm{L}=$ lumen of capillary. Bar, $0.5 \mu \mathrm{m}$
The labelling for HSPG core protein in the GBMs was specific, but did not show any definite distribution (Figs. $3 \mathrm{a}-\mathrm{c}$ ). There was no significant difference in labelling densities for HSPG core protein in glomerular basement membranes among the three groups of animals (Table 2).

All of the animals fed galactose only developed cataracts at the time of killing; most of them had cataracts within four months on the diet. None of the animals on control and galactose-sorbinil diets had cataracts.

Table 2. Labelling densities for macromolecular antigens in glomerular capillary basement membranes in three groups of animals, each composed of five animals (Mean number of gold particles $/ \mu \mathrm{m}^{2}$ basement membrane area $\pm \mathrm{SD}$ )

\begin{tabular}{lcll}
\hline Diet & Collagen IV & Laminin & HSPG core protein \\
\hline Control & $7.59 \pm 1.34$ & $60.53 \pm 10.84$ & $7.79 \pm 3.33$ \\
Galactose & $14.21 \pm 4.64^{\mathrm{a}}$ & $59.20 \pm 11.91^{\mathrm{b}}$ & $5.40 \pm 0.89^{\mathrm{b}}$ \\
Galactose & $12.41 \pm 0.53^{\mathrm{c}, \mathrm{d}}$ & $42.31 \pm 12.04^{\mathrm{e}}$ & $5.59 \pm 2.03^{\mathrm{e}}$
\end{tabular}

+ Sorbinil

a Differs significantly from the control value $(p=0.015) ;{ }^{b}$ No significant difference from either control or sorbinil values; ${ }^{c}$ Differs from the control value $(p<0.001)$; ${ }^{d}$ No significant difference from the galactose value; ${ }^{e}$ No significant difference from the control values. $\mathrm{HSPG}=\mathrm{Heparan}$ sulfate proteoglycan

\section{Discussion}

Our results demonstrate that nine months of experimental galactosaemia in rats for nine months induces GBM thickening as it does in capillaries of the retina $[14,15]$ and the cerebral cortex [16]. Long-term galactosaemia has also been reported to produce similar GBM thickening in dogs, although other typical features of diabetic nephropathy such as mesangial expansion, glomerular obliteration and renal hypertrophy were absent in these animals [18]. In a relatively short-term study (seven weeks), it has been shown that galactose-fed rats develop functional abnormalities, including increases in urine volume, creatinine clearance, urinary albumin excretion and urinary $\mathrm{N}$-acetyl glucosaminidase similar to those seen early in the course of diabetic nephropathy [27].

However, GBM thickening in galactosaemic rats could not be prevented by sorbinil at a dose that prevents thickening of capillary basement membranes in the retina $[14,15,20]$ and the cerebral cortex [16]. Our study is the first demonstration, to our knowledge, that an aldose reductase inhibitor fails to prevent thickening of any microvascular basement membrane in experimental galactosaemia. Recent studies in rats with streptozotocin-induced diabetes have also shown that aldose reductase inhibitors fail to prevent glomerular structural changes including thickened GBMs [11-13]. While one study failed to show 


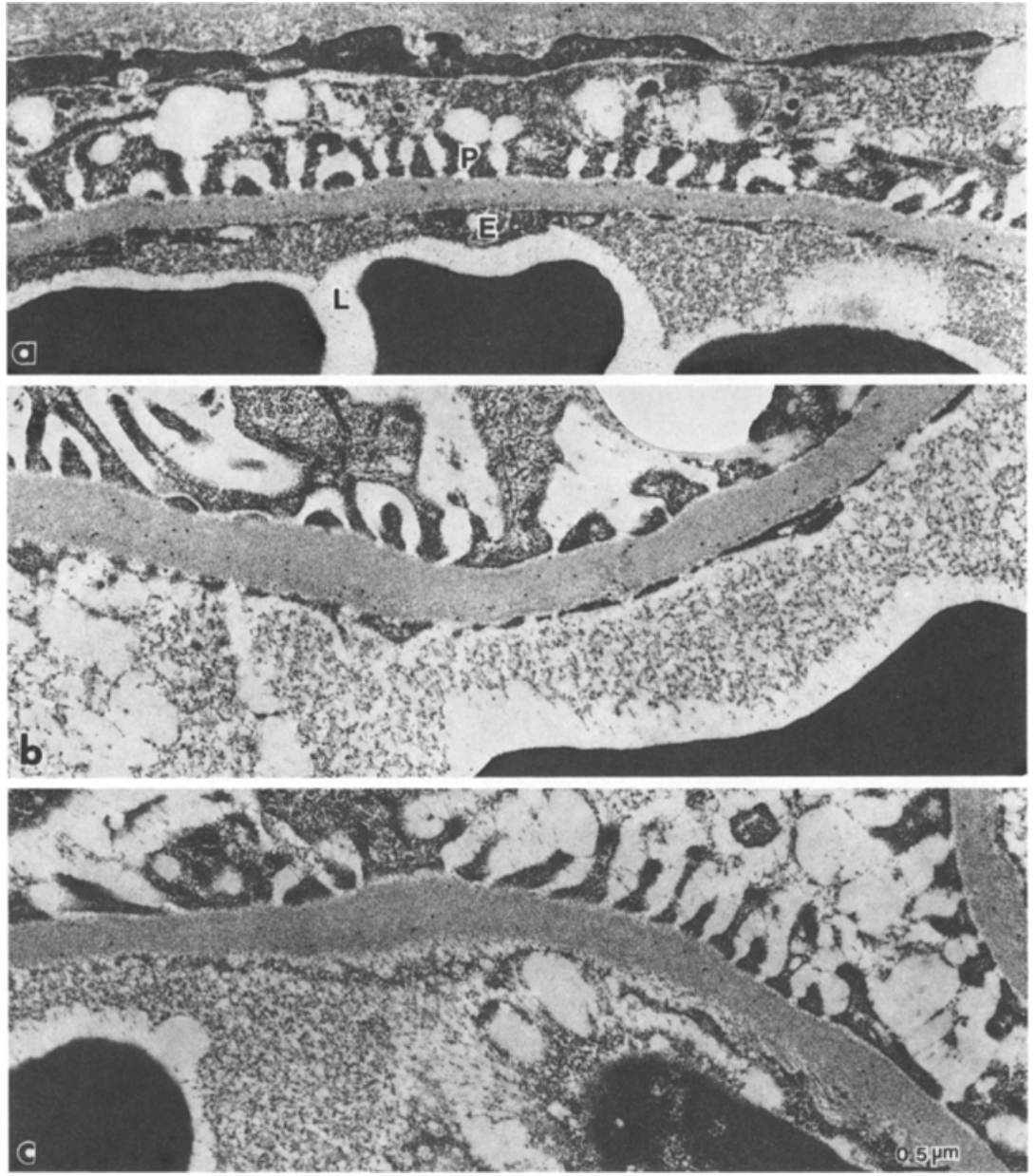

Fig.3a-c. Immunogold labelling for heparan sulfate proteoglycan (core protein) in glomerular basement membranes in rats on a control diet, b galactose diet, c galactose plus sorbinil diet. Antigenic sites are labelled with $15 \mathrm{~nm}$ size gold particles. $\mathrm{P}=$ podocyte; $\mathrm{E}=$ endothelium; $\mathrm{L}=$ lumen of capillary. Bar, $0.5 \mu \mathrm{m}$ any effect of aldose reductase inhibitors on increased mesangial fractional volume in diabetic rats [12], another study demonstrated that these drugs could prevent mesangial expansion in diabetic rats on a high protein diet [11]. Recently, Mauer et al. reported that sorbinil can decrease GBM width in normal rats, although the mechanism is not known [8]. The disparity in effects of aldose reductase inhibitors in different tissues indicates that the polyol pathway may not be involved in the pathogenesis of GBM thickening in galactosaemic rats, even though it appears to have a role in the basement membrane thickening that occurs in the retinal and cerebral cortical capillaries of these animals. Experiments on aldose reductase inhibition and renal haemodynamics have shown mixed results. Administration of sorbinil or myo-inositol partially decreases the elevated glomerular filtration rate in diabetic rats $[7-9,28]$, whereas other investigators were unable to show any effect of aldose reductase inhibition [10] or myo-inositol supplementation [29] on hyperfiltration in diabetic rats.

Our immunogold experiments indicate that the labelling density of collagen type IV in the GBMs of galactosaemic rats is significantly increased $(p=0.015)$ by comparison with the control rats, although there was no change in the labelling density of laminin and HSPG core protein. We have described increases in labelling densities of collagen type IV and laminin in basement membranes in the retinal capillaries of galactosaemic animals [21]. Our results are in agreement with the findings that increased levels of collagens occur in the renal glomeruli of rats in experimental diabetes [30,31]. Kanwar and Farquhar [32] have shown that the array of negatively charged HSPG residues in the GBMs provide an effective filtration barrier, and their loss in glomeruli of diabetic animals [33] and human diabetic subjects [34] might be a major cause for the breakdown of this barrier. However, a different study has shown that there is an increased incorporation of ${ }^{35} \mathrm{SO}_{4}$ into proteoglycan of the basement membrane-producing murine EHS tumour grown at high glucose concentrations [35]. Cohen, et al. have demonstrated undersulphation in GBMs in experimental diabetes, that was independent of the polyol pathway activation [36]. Although we did not find any change in the labelling density of HSPG core protein in our experimental animals, our results do not exclude the possibility that there might be alterations in the degree of sulphation of the heparan sulphate moiety of the HSPG and possibly the number of anionic sites in the GBMs of galactosaemic rats without any change in the concentration of the HSPG core protein.

The immunogold technique used in our study does not permit determination of the absolute concentrations of various components of the GBMs, but it does allow us to compare the relative concentrations of individual compo- 
nents among different groups of experimental animals. The labelling density of antigens in the post-embedding immunogold technique is independent of the thickness of the sections and penetration of antibodies as only those antigenic sites exposed at the surface of the sections react with the antibodies and are visualized as gold particles [37]. As some of the epitopes might be affected during fixation and processing, all tissue samples were fixed, processed and embedded identically, and also sections were stained immunocytochemically in the same way. By using such a standardized protocol, one can effectively compare the relative concentrations of a specific antigen in similar tissue sites [37,38]. That the amount of gold labelling for antigens obtained with the immunogold technique correlates well with the concentrations of antigens estimated with biochemical [39, 40] or RIAs [41] supports the validity of the quantitative electron microscopic immunogold technique.

The precise biochemical mechanisms involved in thickening of the GBMs in diabetes and galactosaemia are not known. It is unclear whether the increase in collagen type IV in thickened basement membranes in capillaries of the kidneys, and the retina [21] in galactosaemic rats is due to increased synthesis or decreased degradation of the basement membrane macromolecules. Increased enzymatic [41] or non-enzymatic [42] glycation that results in post-translational modification of proteins may play an important role in the pathogenesis of GBM thickening in diabetes or galactosaemia. However, other results suggest a direct effect of hexose levels on the genome. Elevated levels of laminin B 1 mRNA have been reported in the kidneys of streptozotocin-induced diabetic rats [43], and increased expression of collagen type IV and fibronectin [44] mRNA have been described in cultured endothelial cells exposed to high glucose concentrations.

In summary, our study demonstrates that an increase in thickness together with an increase in relative concentrations of collagen type IV takes place in the GBMs of galactosaemic rats, but treatment with an aldose reductase inhibitor does not prevent the GBM thickening. The polyol pathway, therefore, seems an unlikely biochemical cause for this lesion. The biochemical mechanism that is responsible for the pathogenesis of these changes in the kidneys of diabetic and galactosaemic animals remains to be determined.

Acknowledgements. This work was supported by RO1 EY-02566 grant from the National Eye Institute, US National Institutes of Health (to RNF); by a grant from the American Diabetes Association, Michigan Affiliate and a postdoctoral fellowship from the Juvenile Diabetes Foundation International (both to AD), and by a departmental unrestricted grant from Research to Prevent Blindness, Inc., New York. We thank Mr. T. Turczyn for technical assistance.

\section{References}

1. Kinoshita JH (1965) Cataracts in galactosemia. Invest Ophthalmol Vis Sci 4: 786-799

2. Gabbay KH (1973) The sorbitol pathway and the complications of diabetes. N Engl J Med 288: 831-836
3. Ludvigson MA, Sorenson RL (1980) Immunohistochemical localization of aldose reductase. II Rat eye and kidney. Diabetes 29: $450-459$

4. Kikkawa R, Umemura K, Haneda M, Arimura T, Ebata K, Shgeta Y (1987) Evidence of existence of polyol pathway in cultured rat mesangial cells. Diabetes 36: 240-243

5. Beyer-Mears AJ, Nicolas-Alexandre J, Cruz E (1983) Sorbinil inhibition of renal aldose reductase. Fed Proc 42: 858

6. Gabbay KH, O'Sullivan JB (1968) The sorbitol pathway in diabetes and galactosemia: enzyme and substrate localization and changes in the kidney. Diabetes 17:300

7. Bank N, Mower P, Aynedjian HS, Wilkes BM, Silverman S (1989) Sorbinil prevents glomerular hyperperfusion in diabetic rats. Am J Physiol 256: F1000-1006

8. Tilton RG, Chang K, Pugliese G, Eades DM, Province MA, Sherman WR, Kilo C, Williamson JR (1989) Prevention of hemodynamic and vascular albumin filtration changes in diabetic rats by aldose reductase inhibitors. Diabetes $37: 1258$ 1270

9. Goldfarb S, Simmons DA, Kern E (1986) Amelioration of glomerular hyperfiltration in acute experimental diabetes by dietary myo-inositol and by an aldose reductase inhibitor. Clin Res 34 [Suppl]: 725 Abstract

10. Daniels BS, Hostetter TH (1989) Aldose reductase inhibition and glomerular abnormalities in diabetic rats. Diabetes 38: 981 986

11. Mauer SM, Steffes MW, Azar S, Brown DM (1989) Effects of sorbinil on glomerular structure and function in long-term diabetic rats. Diabetes 38: 839-846

12. Tilton RG, Pugliese G, Williamson JR (1989) Diabetes-induced glomerular changes in rats are not prevented by Sorbinil. Diabetes 38 [Suppl 2]: 94 Abstract

13. Østerby R, Gundersen HJ (1989) Glomerular basement membrane thickening in streptozotocin diabetic rats despite treatment with an aldose reductase inhibitor. J Diabetic Complications 3: 149-153

14. Frank RN, Keirn RJ, Kennedy A, Frank KW (1983) Galactoseinduced retinal capillary basement membrane thickening: prevention by Sorbinil. Invest Ophthalmol Vis Sci 24: 1519-1524

15. Robison WG Jr, Kador PF, Kinoshita JH (1983) Retinal capillaries: basement membrane thickening by galactosemia prevented with aldose reductase inhibitor. Science 221: 11771179

16. Frank RN, Dutta S, Frank SE (1987) Cerebral cortical capillary basement membrane thickening in galactosaemic rats. Diabetologia 30: 739-744

17. Bank N, Coco M, Aynedjian HS (1989) Galactose feeding causes glomerular hyperperfusion: prevention by aldose reductase inhibition. Am J Physiol 256: F994-999

18. Kern TS, Engerman RL (1987) Kidney morphology in experimental hyperglycemia. Diabetes 36: 244-249

19. Kador PF, Akagi Y, Terubayashi H, Wyman M, Kinoshita JH (1988) Prevention of pericyte ghost formation in retinal capillaries of galactose-fed dogs by aldose reductase inhibitors. Arch Ophthalmol 106: 1099-1102

20. Engerman RL, Kern TS (1989) Retinal vasculature in sorbiniltreated galactosemic dogs. Invest Ophthalmol Vis Sci 30 [Suppl]: 139

21. Das A, Frank RN, Zhang NL, Samadani E (1990) Increases in collagen type IV and laminin in galactose-induced retinal capillary basement membrane thickening - Prevention by an aldose reductase inhibitor. Exp Eye Res 50: 269-280

22. Steffes MW, Brown DM, Basgen JH, Matas AJ, Mauer SM (1979) Glomerular basement membrane thickness following islet transplantation in the diabetic rat. Lab Invest 41 : $116-118$

23. Demarchez M, Hartman DJ, Prunieras M (1987) An immunohistological study of the revascularization process in human skin transplanted onto the nude mouse. Transplantation 43: 896-903

24. Hassell JR, Robey PG, Barrack HJ, Wilczek J, Rennard SI, Martin GR (1980) Isolation of a heparan sulfate-containing proteo- 
glycan from basement membrane. Proc Natl Acad Sci USA 77: 4494-4498

25. Osterby R (1971) Quantitative electron microscopy of the glomerular basement membrane - a methodologic study. Lab Invest $25: 15-24$

26. Steffes MW, Brown DM, Basgen JM, Mauer SM (1980) Amelioration of mesangial volume and surface alterations following islet transplantation in diabetic rats. Diabetes 29: 509-515

27. Lorentz WB, Shihabi ZK, Weidner N (1987) Galactosemic nephropathy in the rat. Clin Physiol Biochem 5:261-267

28. Pugliese G, Speedy A, Tilton RG, Santarelli E, Williamson JR (1988) Effects of myo-inositol supplemented diets on kidney filtration function in rats with streptozotocin diabetes. Diabetes 37 : 244 Abstract

29. Cohen R, MacGregor LC, Spokes KC, Silva P, Epstein FH (1988) Role of myo-inositol in renal Na-K-ATPase changes produced by diabetes. Kidney Int 33 [Suppl]: 372

30. Brownlee M, Spiro RG (1979) Glomerular basement membrane metabolism in the diabetic rats: in vivo studies. Diabetes $28: 121$ 125

31. Hasslacher C, Wahl P (1980) Influence of diabetes control on synthesis of protein and basement membrane collagen in isolated glomeruli of diabetic rats. Res Exp Med 176: 247-253

32. Kanwar YS, Farquhar MG (1979) Anionic sites in the glomerular basement membrane: in vivo and in vitro localization to the laminae rarae by cationic probe. J Cell Biol 81: 137-153

33. Kanwar YS, Rosenzweig LJ, Linker A, Jakubowski ML (1983) Decreased de novo synthesis of glomerular proteoglycans in diabetes: biochemical and autoradiographical evidence. Proc Natl Acad Sci USA 80: 2272-2275

34. Parthasarthy N, Spiro RG (1982) Effect of diabetes on the glycosaminoglycan component of the human glomerular basement membrane. Diabetes 31:738-741

35. Pihlajaniemi T, Myllylä R, Kivirikko KI, Tryggvason K (1982) Effects of streptozotocin diabetes, glucose, and insulin on the metabolism of type IV collagen and proteoglycan in murine basement-forming EHS tumor tissue. J Biol Chem 257: 1491414920

36. Cohen MP, Klepser H, Wu VY (1988) Undersulfation of glomerular basement membrane heparan sulfate in experimental diabetes and lack of correction with aldose reductase inhibition. Diabetes 37: 1324-1327
37. Bendayan M, Nanci A, Kan FWK (1987) Effect of tissue processing on colloidal gold cytochemistry. J Histochem Cytochem 35: 983-996

38. Grant DS, Leblond CP (1988) Immunogold quantitation of laminin, type IV collagen, and heparan sulfate proteoglycan in a variety of basement membranes. J Histochem Cytochem 36: 271-283

39. Posthuma G, Slot JW, Geuze HJ (1984) Immunocytochemical assays of amylase and chymotrypsinogen in rat pancreas secretory granules. J Histochem Cytochem 32: 1028-1034

40. Ratcliffe A, Fryer PR, Hardingham TE (1984) The distribution of aggregating proteoglycans in articular cartilage: comparison of quantitative immunoelectron microscopy with radioimmunoassay and biochemical analysis. J Histochem Cytochem 32: 193-201

41. Spiro RG, Spiro MJ (1971) Studies on the biosynthesis of hydroxylysine-linked disaccharide unit of basement membranes and collagens. III. Tissue and subcellular distribution of glycosyltransferases and the effect of various conditions on the enzyme levels. J Biol Chem 246: 4919-4925

42. Cohen MP, Urdanivia E, Surma M, Wu VY (1980) Increased glycosylation of glomerular basement membrane collagen in diabetes. Biochem Biophys Res Commun 95: 765-769

43. Giambrone MA, Brownlee M (1989) Aminoguanidine treatment normalizes increased steady-state levels of laminin B1 mRNA in kidneys of long-term streptozotocin-induced diabetic rats. Diabetes 38 [Suppl 2]: 83 Abstract

44. Cagliero E, Maiello M, Boeri D, Roy S, Lorenzi M (1988) Increased expression of basement membrane components in human endothelial cells cultured in high glucose. J Clin Invest 82 : $735-738$

Received: 25 October 1989

and in revised form: 2 April 1990

Dr. A. Das

Kresge Eye Institute

Wayne State University School of Medicine

4717 St. Antoine

Detroit, MI 48201

USA 